\title{
Predicting limits of detection in real- time sweat-based human performance monitoring
}

Rudolph, Melanie, Harris, Jonathan, Ratcliff, Erin

Melanie Rudolph, Jonathan K. Harris, Erin L. Ratcliff, "Predicting limits of detection in real-time sweat-based human performance monitoring," Proc. SPIE 11020, Smart Biomedical and Physiological Sensor Technology XVI, 1102000 (2 May 2019); doi: 10.1117/12.2518885

SPIE Event: SPIE Defense + Commercial Sensing, 2019, Baltimore, Maryland, United States 


\title{
Predicting limits of detection in real-time sweat-based human performance monitoring \\ Melanie Rudolph ${ }^{\mathrm{a}}$, Jonathan K. Harris ${ }^{\mathrm{a}}$, Erin L. Ratcliff*a \\ ${ }^{a}$ Department of Materials Science and Engineering, University of Arizona \\ 1235 E. James E. Rogers Way, Tucson, AZ USA 85721
}

\begin{abstract}
Sweat-based human performance monitoring devices offer the possibility of real-time emotional and cognitive awareness in both civilian and military applications. Broad applicability and point of use necessitate non-invasive, printable, flexible, wearable chemical sensors with low power consumption. Sweat fluidics must enable movement of sweat across the sensor compartment within 1 minute to assure only fresh sweat is at the chemical sensor. The sensor material should have reaction kinetics to capture a sufficient number of target molecules for quantification in real-time ( $<1$ minute). Chemical selectivity is critical in complex biofluids such as sweat, which may be comprised of $800+$ biomarkers. Given these constraints, there continues to be significant technological barriers for translation from laboratory-based proof-of-concept demonstrations and scalable manufacturing of devices. Using finite element simulations, we focus on determining which sweat flow geometry and chemical capture dynamics are best suited to meet temporal performance requirements. Two common sensing approaches are compared and contrasted: bio-recognition chemical adsorption events and electrochemical detection. Responsivity of both mechanisms is shown to be highly dependent on fluid dynamics, analyte capture efficiency, analyte concentration, and reaction kinetics. Key metrics of temporal response and capture efficiency will be discussed for a number of state of the art electronic sensor materials, with a focus on the validity of printable platforms.
\end{abstract}

Keywords: Sweat-sensing, Human performance monitoring, predictive simulation, limit of detection, real-time sensing, bio-recognition elements, electrochemical detection

*ratcliff@email.arizona.edu; phone 1520 626-5567; ratcliff.faculty.arizona.edu

\section{INTRODUCTION}

Non-invasive, real-time quantification and qualification of biomarkers in sweat provides direct insight into human operator function and stress. Understanding these processes is critical to improving health through translational biosciences and of high interest to national defense and security. Traditionally, the low concentration of important biomarkers limits the structure of wearable devices, with the common standard being quantification using bulky laboratory benchtop instruments. This practice limits the advancement of real-time health analysis, particularly in situations where biomarkers degrade, such as the enzymatic degradation of peptides. The ideal situation is a low-cost, wearable device where these same capabilities can be used to monitor mental/physical states in real-time, avoiding the degradation of sample transfer to analysis.

In microfluidic devices, transport results from a combination of convection and diffusion. Convection is controlled by the fluid velocity, derived from sweat release rate and the channel geometry while diffusion is the result of a concentration gradient. The gradient is in part controlled by the reaction kinetics specific to the biomarker (for a given solvent) and detection mechanism. The reaction can be a simple adsorption to a surface functionalized with biorecognition elements (BRE), or a chemical or electrochemical reaction with or without catalyst. Accordingly, the relevant reaction parameter could be an adsorption rate constant or a rate constant of an (electro-)chemical or (electrocatalytic reaction.

Cortisol is perhaps the most notable in sweat sensing and has been detected using various approaches ranging from classic competitive immunoassay to simple electrochemical detection to impedance-based detection to sensing with field-effect transistors (FET), condensed into Table 1. Among the approaches discussed here, the lowest limit of detection (LOD) of $1 \mathrm{pg} / \mathrm{mL}$ was found for the FET and impedance sensors. All of the listed sensors used anti-Cortisol 
antibody (Ab) as a BRE in order to improve selectivity. Selectivity towards cortisol was experimentally verified (except in one study) by interference studies using different small-molecule and protein hormones as well as other biomarkers, as relevant to the authors' specific research. In the FET-based sensor, exposure of the Ab-functionalized channel to cortisol in the analyte led to detachment of the $\mathrm{Ab}$ from the channel surface and thereby changed the channel resistance. The FET is distinct from electrochemical and impedance-based sensors, which demonstrate a direct change in current and in capacitance of the channel/analyte interface, respectively, in response to attachment of cortisol from the analyte solution to the $\mathrm{Ab}$ (immunoreaction).

Table 1. Literature review on existing approaches to cortisol sensing and limits of detection (LOD).

\begin{tabular}{|c|c|c|c|}
\hline Sensor Type & Materials System & LOD & Comments on Selectivity \\
\hline $\begin{array}{l}\text { competitive } \\
\text { immunoassay } \\
\text { with } \\
\text { electrochemical } \\
\text { read-out [1] }\end{array}$ & $\begin{array}{l}\text { Pt electrode modified with anti- } \\
\text { cortisol Ab; analyte mixed with } \\
\text { GOx-labeled cortisol conjugate }\end{array}$ & $\begin{array}{l}\mathrm{ca} 1 \\
\mathrm{ng} / \mathrm{mL}\end{array}$ & not studied \\
\hline $\begin{array}{l}\text { electrochemical } \\
\text { [2] }\end{array}$ & $\begin{array}{l}\text { interdigitated Au electrodes on } \\
\mathrm{Si} \text {, modified with anti-cortisol } \\
\text { monoclonal Ab; solution with } \\
\text { redox probe ferricyanide } \\
\text { introduced to chamber after } \\
\text { incubation with analyte (single- } \\
\text { use sensor) }\end{array}$ & $10 \mathrm{pg} / \mathrm{mL}$ & $\begin{array}{l}\text { negligible interference by } \\
\text { epidermal growth factor } \\
\text { receptor, neuron-specific } \\
\text { enolase, and prostate-specific } \\
\text { antigen at } 100 \mathrm{pg} / \mathrm{mL} \text { each }\end{array}$ \\
\hline $\begin{array}{l}\text { electrochemical } \\
\text { [3] }\end{array}$ & $\begin{array}{l}\text { polyaniline/Ag-AgO core-shell } \\
\text { nanoparticle composite } \\
\text { electrode, polyaniline modified } \\
\text { with anti-cortisol } \mathrm{Ab}\end{array}$ & $0.64 \mathrm{pM}$ & $\begin{array}{l}\text { negligible change of sensor } \\
\text { current by } 17-\mathrm{OH} \\
\text { corticosterone and bovine } \\
\text { serum albumin at } 10 \mathrm{ng} / \mathrm{mL} \\
\text { each, but } \mathrm{Ag}-\mathrm{AgO} \text { peak } \\
\text { potentials strongly shifted due } \\
\text { to } 17-\mathrm{OH} \text { corticosterone }\end{array}$ \\
\hline impedance [4] & $\begin{array}{l}\mathrm{ZnO} \text { modified with anti-cortisol } \\
\mathrm{Ab} \text { (used as sweat sensor) }\end{array}$ & $\begin{array}{l}1 \mathrm{pg} / \mathrm{mL} \\
\text { (artificial } \\
\text { sweat) } \\
1 \mathrm{ng} / \mathrm{mL} \\
\text { (human } \\
\text { sweat) }\end{array}$ & $\begin{array}{l}\text { presence of cytokine IL-1 } \beta \\
\text { caused small impedance } \\
\text { change compared to cortisol }\end{array}$ \\
\hline FET [5] & $\begin{array}{l}\text { single-walled carbon nanotubes } \\
\text { modified with anti-cortisol } \\
\text { monoclonal Ab }\end{array}$ & $1 \mathrm{pg} / \mathrm{mL}$ & $\begin{array}{l}\text { no interference by } 21- \\
\text { hydroprogesterone; } \mathrm{Au} \\
\text { contacts treated with } \\
\text { mercaptohexanol to prevent } \\
\text { adsorption of mucin }\end{array}$ \\
\hline
\end{tabular}

From Table 1, it is readily apparent from within the scientific literature that it is very difficult to compare between methodologies. Each detection approach and materials choice results in a different level of sensitivity and performance, including different kinetic reactions and non-standardized experimental conditions. Thus, to move forward in sweat sensing design, simulation of a sensor under various flow conditions and geometries to assess viable solutions is required. The goal of this effort was to develop a mathematical predictive model to guide sensor and the micro-chamber design to determine the structure and flow conditions in the sensor's micro-chamber that enable efficient capture of lowconcentration biomarkers in sweat within 1 minute.

Our approach is to use finite element analysis approximation through the mass transport package of COMSOL. Herein we focus our modeling work on the role of convection (surface or volume flow) of the sweat fluid across a biosensor 
within a microfluidic channel. We considered a range of each input variable to estimate the capture/detection efficiency of each biomarker. We define capture efficiency ( $\eta$; our primary output variable) as:

$$
\text { Capture efficiency }(\eta)=\frac{\frac{\text { molecules detected }}{\text { time } * \text { sensor area }}}{\frac{\text { molecules flow past sesnor }}{\text { time } * \text { sensor area }}}
$$

Herein we focus only on steady-state results to compare between methods.

\section{RESULTS AND DISCUSSION}

\subsection{Effects of geometry, transport parameters, and biomarker concentration with BRE detection scheme.}

We first focus on model on transport and chemical reaction between a biomarker and active sensor area purely by adsorption. Here we target elucidation of the effects of geometry and transport parameters on caption efficiency, as defined in the context of sweat flow velocity $\mathrm{v}(\mathrm{mm} / \mathrm{s})$ and channel geometry, as defined by channel height $\mathrm{H}$. A standard aqueous diffusion coefficient was assumed to describe the transition between convective and diffusive transport $\left(6 \times 10^{-10} \mathrm{~m}^{2} / \mathrm{s}\right)$. The kinetics of the surface reaction were assumed to be controlled by the surface reaction of a classic Langmuir adsorption model

$$
\text { Rate }=k_{\text {adg }} \cdot c_{0} \cdot\left(1-c_{s} / c_{g \max }\right)-k_{\text {des }} \cdot c_{s} / c_{\text {gmax }}
$$

where $\mathrm{k}_{\mathrm{ads}}$ is the rate parameter for adsorption $\left(\mathrm{m}^{3} / \mathrm{s} \bullet \mathrm{mol}\right), \mathrm{k}_{\mathrm{des}}$ is the rate parameter for desorption $\left(\mathrm{s}^{-1}\right), \mathrm{c}_{0}$ is the concentration of the biomarker in solution (bulk, $\mathrm{g} / \mathrm{mL}$ or $\mathrm{mol} / \mathrm{m}^{3}$ ), $\mathrm{c}_{\mathrm{s}}$ is the concentration of sites that contain the adsorbed biomarker and $\mathrm{c}_{\mathrm{s}, \max }$ is the maximum concentration of adsorption sites on the active sensor area within the microfluid channel $\left(\mathrm{mol} / \mathrm{m}^{2}\right)$. Simulation results were based on a fixed initial concentration of $5.3 \times 10^{-9} \mathrm{~mol} / \mathrm{m}^{3}$. The rate constants of adsorption $\left(\mathrm{k}_{\mathrm{ads}}\right)$ and desorption $\left(\mathrm{k}_{\mathrm{des}}\right)$ and the surface site concentration $\left(\mathrm{c}_{\mathrm{s}, \max }\right)$ were also allowed to vary. Table 2 shows the effect of the $\mathrm{k}_{\mathrm{ads}}$ and $\mathrm{k}_{\mathrm{des}}, \mathrm{c}_{\mathrm{s}, \max }$, and $\mathrm{H}$ on capture efficiency. Effectively, $\mathrm{c}_{0}$ concentration is determined by the sweat from each pore, while the other parameters could theoretically be controlled via sample design (site density) and tailoring the adsorption chemistries of the BRE.

\begin{tabular}{|c|c|c|c|c|}
\hline $\begin{array}{c}\mathbf{k}_{\text {ads }} / \\
\mathbf{m}^{3 /(\mathbf{m o l} \cdot \mathbf{s})} \\
\end{array}$ & $\mathbf{k}_{\text {des }} / \mathbf{s}^{-1}$ & $\mathrm{c}_{\mathrm{s}, \max } / \mathrm{mol} / \mathrm{m}^{2}$ & $\mathrm{H} / \mathbf{m m}$ & $\eta / \%$ \\
\hline $5 \cdot 10^{-4}$ & $5 \cdot 10^{-6}$ & $3 \cdot 10^{-8}$ & 0.1 & $2 \cdot 10^{-4}$ \\
\hline $5 \cdot 10^{-2}$ & $5 \cdot 10^{-6}$ & $3 \cdot 10^{-8}$ & 0.1 & $2 \cdot 10^{-2}$ \\
\hline $5 \cdot 10^{-4}$ & $5 \cdot 10^{-10}$ & $3 \cdot 10^{-8}$ & 0.1 & $2 \cdot 10^{-4}$ \\
\hline $5 \cdot 10^{-4}$ & $5 \cdot 10^{-6}$ & $3 \cdot 10^{-6}$ & 0.1 & $2 \cdot 10^{-2}$ \\
\hline $5 \cdot 10^{-4}$ & $5 \cdot 10^{-6}$ & $3 \cdot 10^{-8}$ & 0.01 & $2 \cdot 10^{-3}$ \\
\hline $5 \cdot 10^{-2}$ & $5 \cdot 10^{-6}$ & $3 \cdot 10^{-6}$ & 0.01 & 18.6 \\
\hline $5 \cdot 10^{-2}$ & $5 \cdot 10^{-10}$ & $3 \cdot 10^{-4}$ & 0.01 & 98.4 \\
\hline 5 & $5 \cdot 10^{-10}$ & $3 \cdot 10^{-4}$ & 0.01 & 98.7 \\
\hline
\end{tabular}

Table 2. Effect of sensor parameters on capture efficiency. Bold reflects changes in variable relative to first row.

Figure 1 below shows the distribution of the convective region (red) and the diffusive gradient, as a function of velocity flow rates. We readily observe the expected transport phenomenon that at lower velocity, the diffusive region is 
significantly larger. We also note that as the velocity increases, the diffusive region i) is significantly smaller and ii) becomes asymmetric across the sensor surface. Even though the faster sweat flow increases the surface concentration of biomarker molecules adsorbed to the active sensor area after a given time period, the overall capture efficiency is decreased with higher velocity (as compared in Table 2). Thus, one of our first design guidelines in effective sensor design is to maximize the diffusion layer thickness to promote binding of the biomarker.

In addition to altering the flow velocity, the relative diffusion layer thickness (with respect to the overall channel thickness) can be increased by simply decreasing the channel height. The plot in Figure 2 below compares the effect of the channel height for a subset of the velocity profiles in Figure 1. As one would expect from a classic convective to diffusive transition, decreasing the channel height by a factor of 10 from $100 \mu \mathrm{m}$ to $10 \mu \mathrm{m}$ results in a 10 -fold increase in capture efficiency. However, we note that the current simulation assumes the basic transport mechanism of flow is not changed with channel height. For example, non-specific binding of the biomarker molecules to the walls of the chamber would result in lower capture efficiency that would be more prevalent in smaller flow channels, where the wall surface area to channel volume is increased. While beyond the scope of this specific effort, such an effect could be further explored in future work.

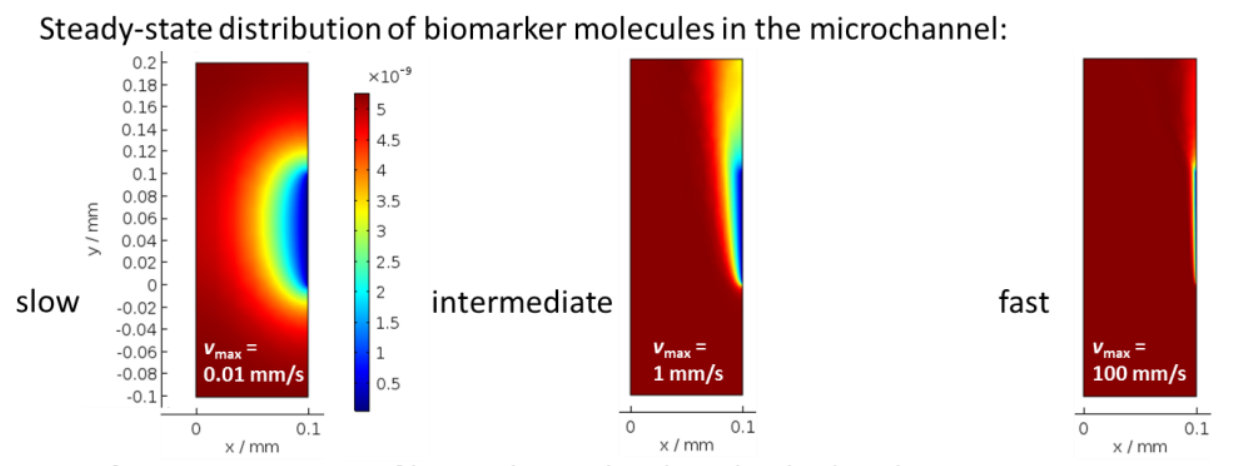

Surface concentration of biomarker molecules adsorbed to the active sensor area:
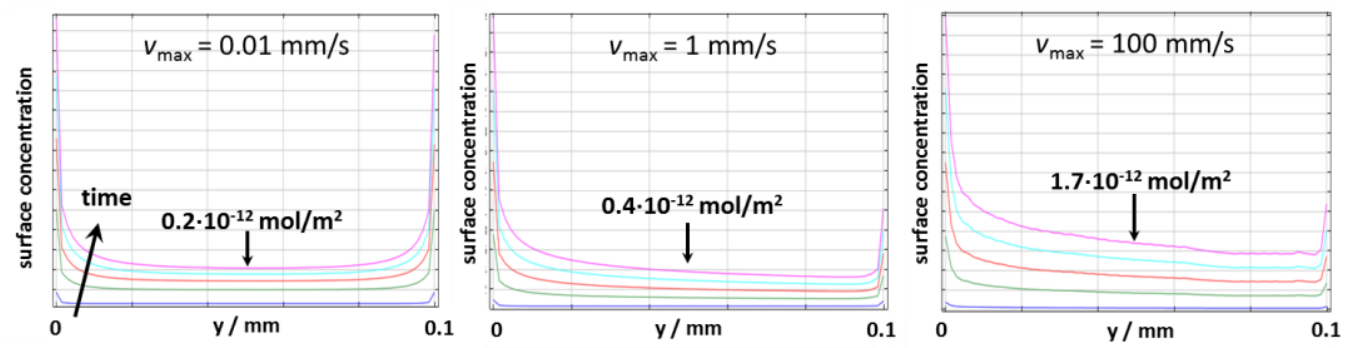

Figure 1. Effect of sweat flow velocity with adsorption detection.

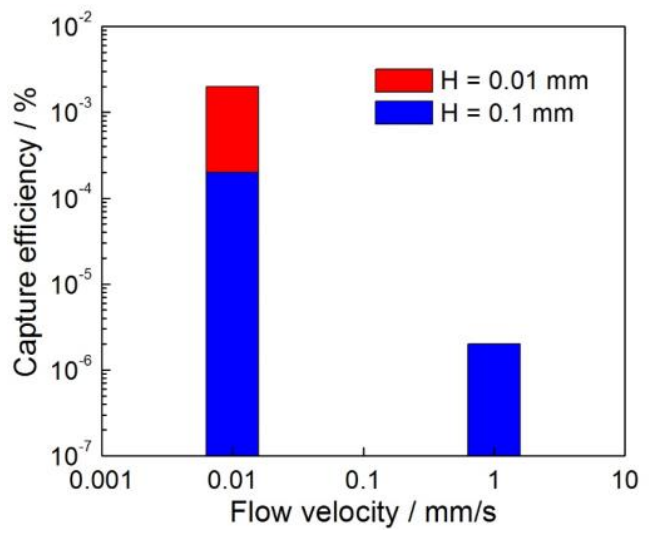

Figure 2. Combined effect of channel height and sweat flow velocity on capture efficiency using a BRE detection strategy. 


\subsection{Finite element analysis simulation (effects of geometry, transport parameters, biomarker concentration) with electrochemical detection.}

Many sensors are based on electron transfer events between biomarkers of interest or passive detection - such as enzymatic generation of peroxide from glucose. As such we additionally focused on the transport and chemical reaction between a biomarker and active sensor area purely by an oxidation-reduction (redox) reaction. Herein we utilized the optimized parameters provided in the BRE study above and directly compare the two mechanisms on steady-state capture efficiency $(\eta)$. A standard diffusion coefficient was assumed to describe the transition between convective and diffusive transport, kinetics were assumed to be controlled by the surface reaction the Butler-Volmer model and only considered faradaic (electron transfer) contributions to current (rate of reaction):

$$
\text { rate }=k_{f} C_{0 x}-k_{b} C_{R}
$$

where $\mathrm{k}_{\mathrm{f}}$ and $\mathrm{k}_{\mathrm{b}}$ are the forward and reverse rate constants for oxidation $(\mathrm{cm} / \mathrm{s})$ and $\mathrm{C}_{\mathrm{ox}}$ and $\mathrm{C}_{\mathrm{R}}$ are the concentrations for the oxidized and reduced species, respectively. Both $\mathrm{k}_{\mathrm{f}}$ and $\mathrm{k}_{\mathrm{b}}$ can be written in terms of three parameters: $\mathrm{k}^{0}$, the standard rate constant $(\mathrm{cm} / \mathrm{s}) ; \alpha$, the symmetry coefficient (unitless with values from 0 to 1 ); and $\mathrm{E}$, the over-potential relative to the standard oxidation potential of the molecule $(\mathrm{V})$ :

$$
\begin{aligned}
& k_{f}=k^{0} \exp \left[-\frac{\alpha E}{k T}\right] \\
& k_{b}=k^{0} \exp \left[\frac{(1-\alpha) E}{k T}\right]
\end{aligned}
$$

Effectively, the Butler-Volmer model allows for a description of a non-equilibrium reaction between the electrode and the biomarker. By changing the over-potential E, one can effectively control the forward and reverse reaction rate constants, and thus drive the reaction to increase sensing efficiency (improved kinetics, relative to mass transport). Such control is unique relative to the Langmuir adsorption isotherm, which requires the use of an equilibrium controlled reaction between the adsorbate molecule and the empty site. Table 3 summarizes the collective effects on capture efficiencies using initial concentration of $5.3 \times 10^{-9} \mathrm{~mol} / \mathrm{m}^{3}, \mathrm{D}=6 \times 10^{-10} \mathrm{~m}^{2} / \mathrm{s}$ and $\mathrm{v}_{\max }$ of $0.01 \mathrm{~mm} / \mathrm{s}$.

\begin{tabular}{|c|c|c|c|c|}
\hline $\mathrm{k}^{0} / \mathrm{cm} / \mathrm{s}$ & $\alpha$ & $\mathrm{E} / \mathrm{V}$ & $\mathrm{H} / \mathrm{mm}$ & $\eta / \%$ \\
\hline $1 \cdot 10^{-3}$ & 0.5 & 0.25 & 0.1 & 83.7 \\
\hline $1 \cdot 10^{-1}$ & 0.5 & 0.25 & 0.1 & 84.1 \\
\hline 1 & 0.5 & 0.25 & 0.1 & 84.1 \\
\hline $1 \cdot 10^{-3}$ & 0.3 & 0.25 & 0.1 & 84.1 \\
\hline $1 \cdot 10^{-3}$ & 0.5 & 0.55 & 0.1 & 84.1 \\
\hline $1 \cdot 10^{-3}$ & 0.5 & 0.25 & 0.01 & 98.7 \\
\hline $1 \cdot 10^{-1}$ & 0.5 & 0.25 & 0.01 & 99.95 \\
\hline
\end{tabular}

Table 3. Effect of sensor parameters on capture efficiency. Bold reflects changes in variable relative to first row. Bold reflects changes in variable relative to first row.

Figure 3A shows the biomarker capture efficiency as a function of over-potential $\mathrm{E}$ and Figure $3 \mathrm{~B}$ shows $\eta$ as a function of the standard rate constant $\mathrm{k}^{0}$. In Figure $3 \mathrm{~A}$, the rate constant was held constant at $1 \times 10^{-3} \mathrm{~cm} / \mathrm{s}$ based on the measured standard rate coefficient of many small, redox-active molecules undergoing charge transfer to metal electrodes. The over-potential was varied in the simulation, from driving the reduction reaction (negative over-potential) to driving the oxidation reaction (positive over-potentials). The results demonstrate the ability of the researcher to readily explore and control the rate of the kinetic mechanism at the electrode surface, independent of mass transport controlled by the velocity. With very little change in the over-potential, the reaction readily reaches a saturation of capture efficiency of $\sim 84 \%$, which is indicative of a mass transport limit, not a kinetic limit.

Confirmation of a mass transport limit is visible in the bottom plot in Figure 3B, where the $\mathrm{k}^{0}$ was hypothetically varied from $10^{-6}$ to $10^{0} \mathrm{~cm} / \mathrm{s}$ while keeping the over-potential constant $(0.25 \mathrm{~V})$. Only at very low rate constants do we observe a 
deviation in the capture efficiency from $84 \%$. Furthermore, by increasing the diffusion coefficient in this virtual experiment, we see a capture efficiency reaching $100 \%$, where mass transport is able to keep up with ultrafast kinetics. We indicate here that the results in Figure 3B are purely a theoretical construct for confirmation of the mass transport limit- varying the standard rate constant this much would require a significant materials and electrode engineering effort.
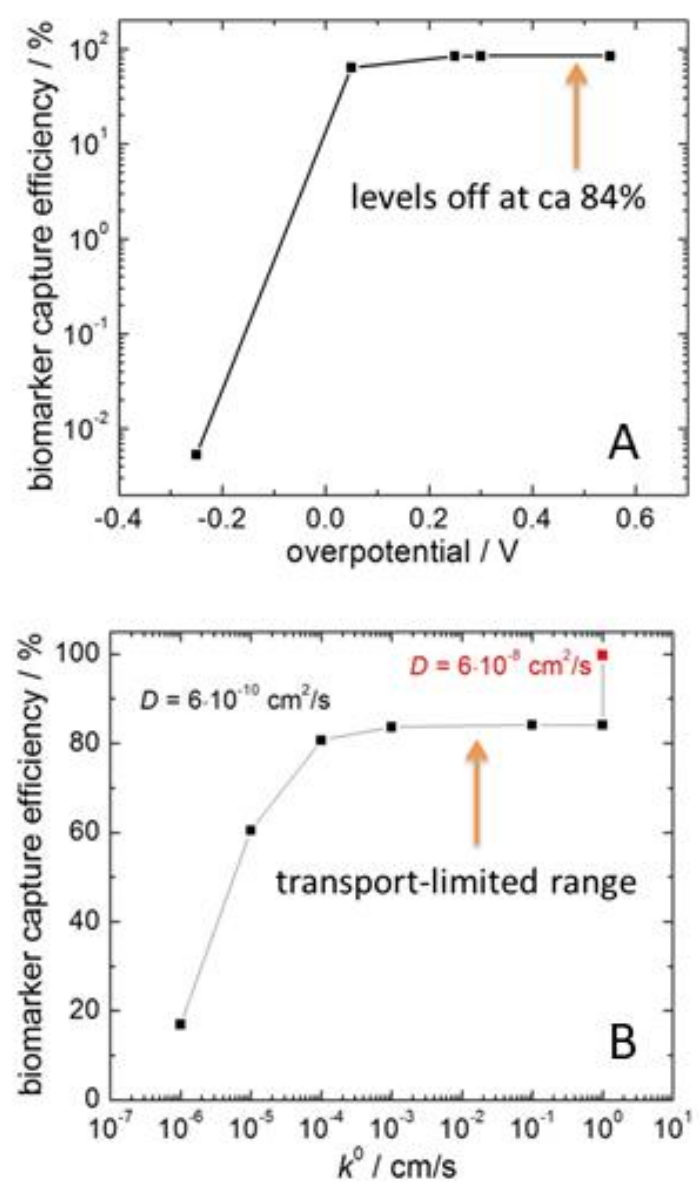

Figure 3. Biomarker capture efficiency as a function of (A) electrochemical over-potential and (B) standard rate constant.

For direct comparison with the BRE detection results presented in Figures 1 and 2, the sweat flow velocity (v) was also varied; with the results provided in Figure 4. Increasing the convective flow rate through the microfluidic channel increases the rate of the transport-limited electrochemical reaction simply because there is more available reactant present at the electrode surface. However, as with the BRE approach, the capture efficiency is strongly reduced with increasing $\mathrm{v}$ because more molecules flow past the active sensor area without reacting. Similarly, maintaining a relatively low sweat flow rate $\left(\mathrm{v}_{\max } \sim 0.01 \mathrm{~mm} / \mathrm{s}\right)$ allows for higher capture efficiency, although the difference in the two approaches is readily apparent in Table 4 . The BRE was only able to achieve a $\eta \sim 10^{-4} \%$, while the electrochemical approach resulted in an $\eta \sim 84 \%$. Decreasing the channel height $\mathrm{H}$ from 0.1 to $0.01 \mathrm{~mm}$ resulted in an increase in the capture efficiency of the BRE approach to $\sim 10^{-3} \%$, while the electrochemical approach reached a $100 \%$ capture efficiency with a similar reduction in channel height to $0.01 \mathrm{~mm}$ (Table 4). From Table 4, we conclude that using an electrochemical detection scheme is ideal in the case of redox active species and is useful as it allows for a reduction in the sweat volume needed to accurately determine the concentration. For reference, a $1 \mathrm{~nL}$ volume is equivalent to a droplet radius of $\sim 80$ microns, which is sufficient to fill a sweat channel height of $10 \mu \mathrm{m}$ provided in Table 4 and represents sweat collection from a single pore. 

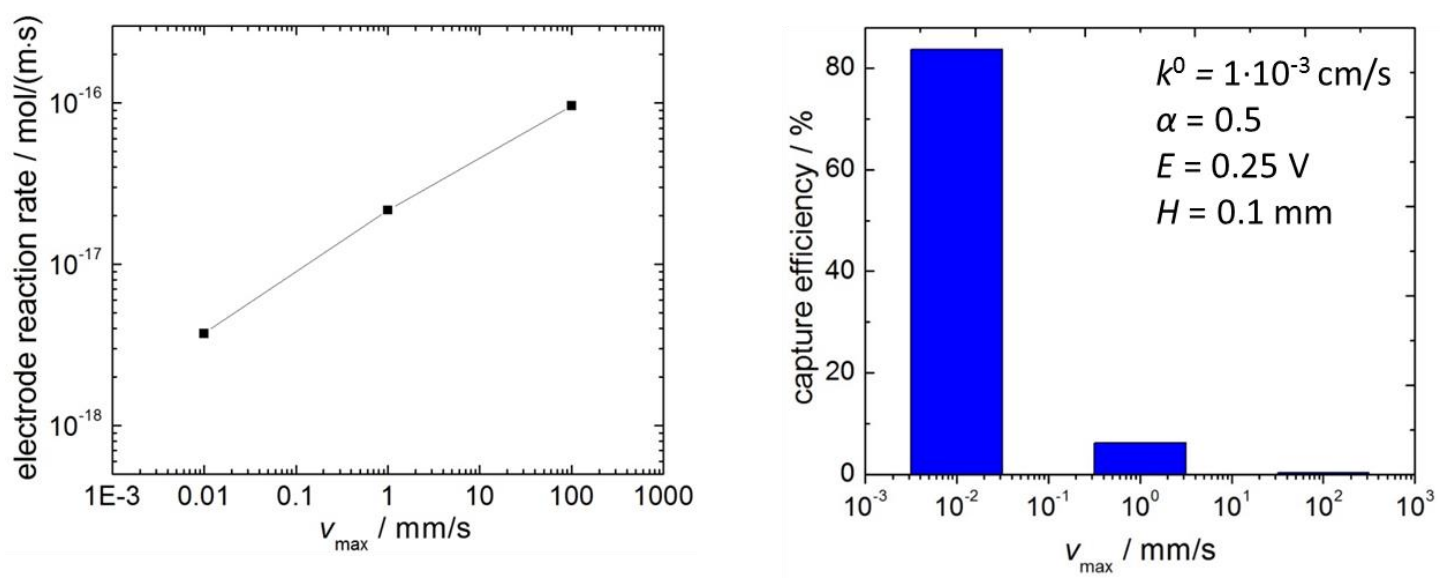

Figure 4. Effect of sweat flow rate on biomarker capture efficiency with electrochemical detection.

Table 4. Capture efficiency $(\eta)$ maximum as a function of detection scheme.

\begin{tabular}{|l|l|l|l|}
\hline Detection mechanism & $\mathbf{V}_{\max } / \mathbf{m m} / \mathbf{s}$ & $\mathbf{H} / \mathbf{m m}$ & $\mathbf{\eta} / \mathbf{\%}$ \\
\hline $\mathrm{BRE}^{\mathrm{a}}$ & 0.01 & 0.1 & $2 \cdot 10^{-4}$ \\
\hline $\mathrm{BRE}$ & 0.01 & 0.01 & $2 \cdot 10^{-3}$ \\
\hline Electrochemical $^{\mathrm{b}}$ & 0.01 & 0.1 & 83.7 \\
\hline Electrochemical $^{\mathrm{a}}$ & 0.01 & 0.01 & 98.7 \\
\hline
\end{tabular}

${ }^{\mathrm{a}} \mathrm{k}_{\mathrm{ads}}=5 \cdot 10^{-4} \mathrm{~m}^{3} / \mathrm{mol} \cdot \mathrm{s}, \mathrm{k}_{\mathrm{des}}=5 \cdot 10^{-6} \mathrm{~s}^{-1}, \mathrm{c}_{\mathrm{s}, \max }=3 \cdot 10^{-8} \mathrm{~mol} / \mathrm{m}^{2}$

${ }^{\mathrm{b}} \mathrm{k}_{0}=1 \cdot 10^{-3} \mathrm{~cm} / \mathrm{s}, \alpha=0.5, \mathrm{E}=0.25$.

\section{CONCUSIONS}

Development of human performance monitoring devices to quantify sweat biomarkers necessitates non-invasive, realtime monitoring of ultra-low concentrations of hormones, proteins, and neurotransmitters. Broad applicability and point of use necessitate non-invasive, printable, flexible, wearable chemical sensors with low power consumption. This work presents finite element simulations of two prominent detection strategies for microfluidic devices: chemical adsorption and electrochemical detection. This preliminary work focused on changes in experimental parameters on the sensor chamber, with electrochemical detection far out-performing BRE approaches at single sweat pore levels.

\section{FUNDING ACKNOWLEGEMENTS}

This material is based on research sponsored by Air Force Research Laboratory under agreement number F A8650-13-2731 1. The U.S. Government is authorized to reproduce and distribute reprints for Government purposes notwithstanding any copyright notation thereon. The views and conclusions contained herein are those of the authors and should not be interpreted as necessarily representing the official policies or endorsements, either expressed or implied, of Air Force Research Laboratory or the U.S. Government. A portion of this work was supported by the Defense and Security Research Institute through the Technology and Research Initiative FUND (TRIF) of Arizona.

\section{REFERENCES}

[1] M. Yamaguchi, Y. Matsuda, S. Sasaki, M. Sasaki, Y. Kadoma, Y. Imai, D. Niwa, V. Shetty, "Immunosensor with fluid control mechanism for salivary cortisol analysis", Biosensors \& Bioelectronics 41, 186-191 (2013).

[2] A. Vasudev, A. Kaushik, Y. Tomizawa, N. Norena, S. Bhansali, "An LTCC-based microfluidic system for label-free, electrochemical detection of cortisol”, Sensors and Actuators B-Chemical 182, 139-146 (2013). 
[3] A. Kaushik, A. Vasudev, S.K. Arya, S. Bhansali, "Mediator and label free estimation of stress biomarker using electrophoretically deposited Ag@AgO-polyaniline hybrid nanocomposite", Biosensors \& Bioelectronics 50, 35-41 (2013).

[4] R.D. Munje, S. Muthukumar, A.P. Selvam, S. Prasad, "Flexible nanoporous tunable electrical double layer biosensors for sweat diagnostics", Scientific Reports 5 (2015).

[5] C. Tlili, N.V. Myung, V. Shetty, A. Mulchandani, "Label-free, chemiresistor immunosensor for stress biomarker cortisol in saliva", Biosensors \& Bioelectronics 26(11) 4382-4386 (2011). 